\title{
Draft Genome Sequence of Acinetobacter Y1, a Heterotrophic Nitrifying and Aerobic Denitrifying Bacterium Isolated from Coke Plant Wastewater
}

\author{
Ting YANG ${ }^{1, a}$, Ya YANG ${ }^{1, b}$, Yu-xiang LIU ${ }^{1, c, *}$ and Ze-ying LIU ${ }^{2, d}$ \\ ${ }^{1}$ Taiyuan University of Technology, College of Environmental Science and Engineering \\ ${ }^{2}$ Shanxi Key Lab of Coal Science and Technology \\ Shanxi, China

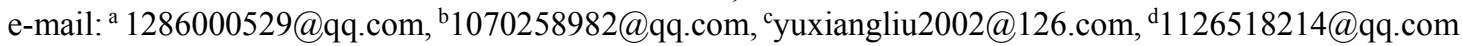

\begin{abstract}
Acinetobacter sp. Y1, with a remarkable capability of heterotrophic nitrification and aerobic denitrification, was isolated from the activated sludge of a coke plant wastewater. It can simultaneously remove specific organic matter and different nitrogen (ammonium nitrogen, nitrite nitrogen and nitrate nitrogen). For further study of the mechanism of nitrogen removal, its draft genome was sequenced. Results show that the 3, 384, 069 bp draft genome consists of 24 scaffolds with 3173 protein-coding sequences, 63 tRNAs and 8 rRNAs. Of the total protein-coding sequences, $99.15 \%$ had hits in the NR database. KEGG database annotation results indicate 1708 genes involved in 167 metabolic pathways and possessed the genes of degrading many types of organic chemicals. Multiple genes relating to nitrogen metabolism and degrading many types of organic chemicals were annotated.
\end{abstract}

Keywords-Genome sequence, Acinetobacter, Nitrogen metabolism, Heterotrophic nitrification, Aerobic denitrification

\section{INTRODUCTION}

Most of the member of the genus Acinetobacter has been reported as clinical isolates[1-3]. Only a few strains of the family have been reported to be able to metabolize a diverse range of compounds [4-7]. In recent years, some isolates within the genus proved to be heterotrophic nitrifier, such as Acinetobacter junii $\mathrm{YB}[8]$, Acinetobacter calcoaceticus HNR[9], Acinetobacter sp. HA2[10], Acinetobacter sp. Y16[11]. Most of them can also simultaneously denitrify their nitrification products to nitrogenous gas under aerobic condition. Heterotrophic nitrification-aerobic denitrification has attracted increasing attention because it possesses the merit that traditional nitrogen removal process can't compare with. The two possible pathway of nitrogen metabolism was suggested, one is similar to autotrophic nitrification process, in which ammonium is firstly converted to hydroxylamine, followed by hydroxylamine oxidation to nitrite and/or nitrate, and then nitrification products is denitrify to nitrogenous gas[8], while the other one is denitrification via hydroxylamine rather than nitrite or nitrate $[12,13]$. However, it is still difficult to generalize the actual metabolic mechanisms of coupling of heterotrophic nitrification and aerobic denitrification just depending on the detection of intermediate products, end products, individual enzymes and related genes. Researches of heterotrophic nitrifier always refer to autotrophic one due to the limitation of the species number. Hitherto, some phenomena in the nitrogen removal of heterotrophic nitrification and aerobic denitrification can't explain, such as the heterotrophic nitrogen removal ability of different bacteria depends on different carbon sources[14]; Alkaline environment is conducive to heterotrophic nitrogen removal in bacteria, but reported fungi prefer acidic condition[15].

Acinetobacter sp. Y1 showed the remarkable capacity of combined heterotrophic nitrification-aerobic denitrification[16]. It can remove specific organic matter and different nitrogen (ammonium nitrogen, nitrite nitrogen and nitrate nitrogen) simultaneously [17]. Study on the genome sequence of the strain might provide useful insight into its characteristics of nitrogen and carbon metabolism, which will stimulate and facilitate further studies for heterotrophic nitrification and aerobic denitrification.

\section{MATERIALS AND METHODS}

\section{A. Cultivation of Acinetobacter sp. Y1}

Acinetobacter sp. Y1 was isolated from the activated sludge of a coke plant wastewater located in Shanxi Province, North China and was preserved in Shanxi Key Lab of Coal Science and Technology, Taiyuan University of Technology.

\section{B. DNA Extraction, Library Construction, and Sequencing}

Genomic DNA was extracted from the 24-h culture using a Rapid Bacterial Genomic DNA Isolation Kit (Sangon Biotech.Co.,Ltd.Shanghai, China) and stored in Elution Buffer at $-20{ }^{\circ} \mathrm{C}$. Libraries were generated with VAHTSTM Nano DNA Library Prep Kit for Illumina ${ }^{\circledR}$.

The genome of Acinetobacter Y1 was sequenced using the Illumina Miseq platform with a PE300 strategy. Clean reads were assembled using SPAdes and velvet, while remaining gaps were tried to be closed by GapCloser, GapFiller, and corrected by PrlnSes-G. Protein-coding sequences (CDSs) were predicted using the NCBI Prokaryotic Genome Annotation Pipeline (PGAP) (http:/www.ncbi.nlm.nih.gov/genomes/static/Pipeline.html), and rapid annotation using Subsystem Technology (RAST) version 2.0[18], and then matched with the databases of PFAM, NR, CDD (Conserved Domains Database), SwissProt, COG, TrEMBL, GO, KEGG. The tools of RNAscanSE and RNAmmer were used to annotate tRNA and rRNA genes, respectively[19, 20]. 


\section{RESUlTS}

The properties and the statistics of the genome of Acinetobacter sp. Y1 are summarized in Table I. The assembly of the draft genome sequence of Acinetobacter Y1 was consisted of 24 scaffolds amounting to $3384069 \mathrm{bp}$. The $\mathrm{G}+\mathrm{C}$ content was $39.56 \%$. No plasmid was found. A sum of 3173 CDSs with an average length of $932.04 \mathrm{bp}$ was detected in the genome. Moreover, 63 tRNAs and 8 rRNAs were identified.

Different databases about nucleotide sequence or amino acid sequence have different focus. Of the total CDSs, $99.15 \%$ had hits in the NR database, followed by $98.55 \%$ in TrEMBL, the lowest ratio $53.83 \%$ in KEGG. The annotated genes in KEGG, Swiss-Prot and COG databases could also be assigned in the NR database, which may provide the valuable information of Acinetobacter Y1. Among the CDSs, $2514(79.23 \%)$ proteins could be assigned to clusters of orthologous groups based on the analysis of CDD. COG database annotation got 2295 genes, corresponding to 20 COG functional categories. The distribution of genes into COG functional categories is depicted in Fig.1. The most abundant COG category was "General function prediction only"(13.51\%), followed by "Function unknown" (10.37\%), "Amino acid transport and metabolism"(7.63\%), "Transcription"(7.45\%), "Translation, ribosomal structure and biogenesis" $(7.32 \%)$. For GO functional classification annotation, 2113 genes were annotated to three terms: biological process, cellular component and molecular function (Fig.2).

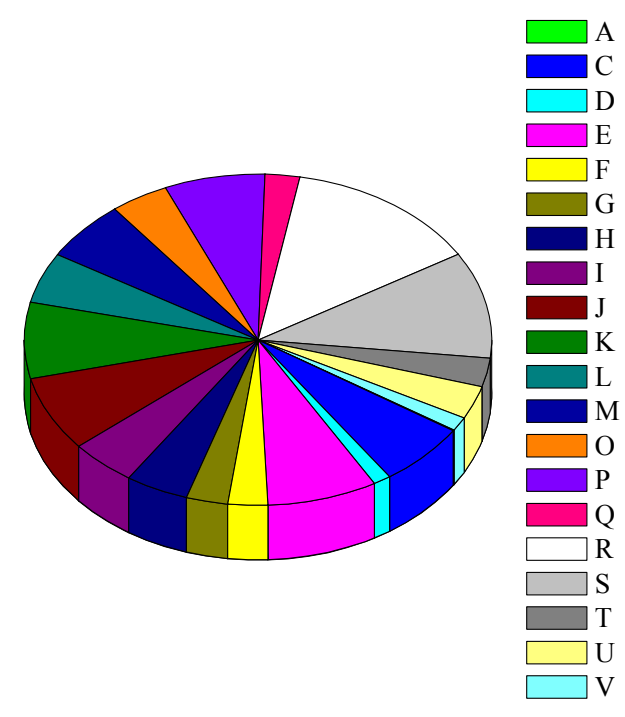

Figure 1. Secondary function classification of COG

Note: A, RNA processing and modification; C, Energy production and conversion; D, Cell cycle control, cell division, chromosome partitioning; E, Amino acid transport and metabolism; F, Nucleotide transport and metabolism; G, Carbohydrate transport and metabolism; H, Coenzyme transport and metabolism; I, Lipid transport and metabolism; J, Translation, ribosomal structure and biogenesis; K, Transcription; L, Replication, recombination and repair; M, Cell wall/membrane/envelope biogenesis; O, Posttranslational modification, protein turnover, chaperones; P, Inorganic ion transport and metabolism; Q, Secondary metabolites biosynthesis, transport and catabolism; R, General function prediction only; S, Function unknown, T, Signal transduction mechanisms; U, Intracellular trafficking, secretion, and vesicular transport; V, Defense mechanisms. 


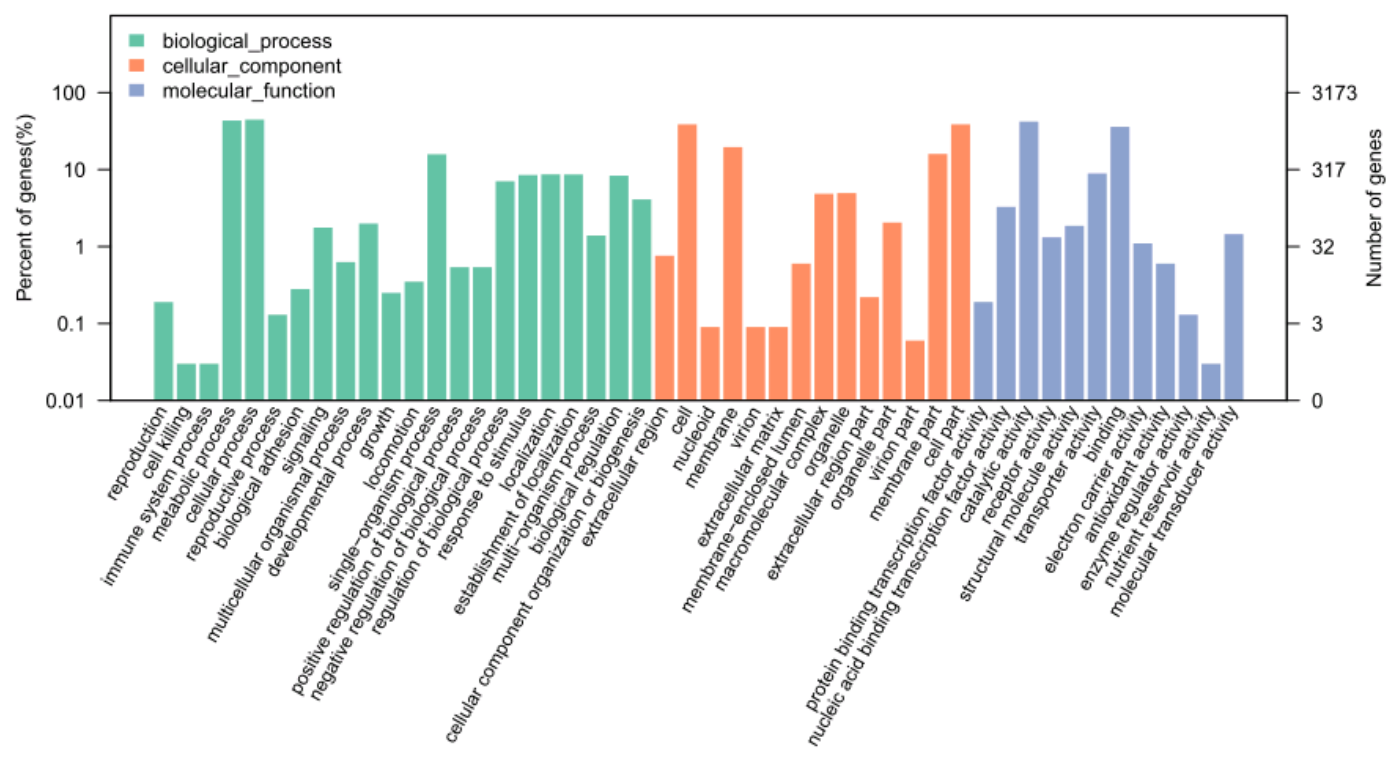

Figure 2. GO classification

KEGG database annotation results indicated 1708 genes involved in 167 metabolic pathways (Table II). Besides the general metabolic pathways, such as citrate cycle and glycolysis/gluconeogenesis metabolism, Acinetabacter sp. Y1 possessed the capacity of methane metabolism, pyruvate metabolism, starch and sucrose metabolism, fructose and mannose metabolism, C5-branched dibasic acid metabolism, sulfur metabolism, glyoxylate and dicarboxylate metabolism. Although the strain possessed glycolysis, citrate cycle and sucrose metabolism, it couldn't effectively removal ammonium when glucose/sucrose was used as sole carbon source in our medium[17]. Heterotrophic nitrification is integrated into metabolisms, particularly the metabolism of special carbon source. The role of carbon in heterotrophic nitrification need be investigated further.

In the study, multiple genes relating to nitrogen metabolism were annotated. In combined heterotrophic nitrification-aerobic denitrification, the possible nitrogen removal pathway is the conversion of ammonium to hydroxylamine is catalyzed by ammonia monooxygenase(AMO), and followed by hydroxylamine oxidation to nitrite by hydroxylamine oxidoreductase (HAO), nitrate reductase (NR) and nitrite reductase (NiR) catalyze the reduction of nitrate to nitrite and nitrite to nitrogenous gas respectively. HAO, a key enzyme in nitrification, has been purified from various organisms including autotrophic and heterotrophic nitrifier. It has been reported that HAOs are distinct from different organisms. HAO from $\mathrm{P}$. denitrificans is a small periplasmic monomer $(20-\mathrm{kDa})$ containing ferric iron[21], while the enzyme from Pseudomonas PB16 is a homo-dimer of $68-\mathrm{kDa}$ subunits with no detectable cofactors[22]. In the present study, hydroxylamine oxidoreductase gene was not identified. However, the HAO of $47-\mathrm{kD}$ has been purified from Acinetobacter sp. Y1 in our previous study. The HAO in
Acinetobacter sp. Y1 may be distinct from others. Further study should be conducted to confirm the HAO gene in the strain.

Furthermore, Acinetabacter sp. Y1 was analysised to possess the genes of degrading many types of organic chemicals, such as ethylbenzene, styrene, naphthalene, limonene and pinene, chlorocyclohexane and chlorobenzene, aminobenzoate, ketone bodies, nitrotoluene, polycyclic aromatic hydrocarbon, benzoate, toluene, bisphenol, geraniol, aromatic compounds, dioxin, fluorobenzoate, xylene, chloroalkane and chloroalkene, atrazine, caprolactam. The results indicate that Acinetabacter sp. Y1 is a promising candidate for future application in industry wastewater.

TABLE I. GENERAL FEATURES OF ACINETOBACTER SP. Y1 DRAFT GENOME

\begin{tabular}{|c|c|}
\hline Class & Number \\
\hline Size $(\mathrm{bp})$ & 3384069 \\
\hline G+C content $(\%)$ & 39.56 \\
\hline CDSs & 3173 \\
\hline Min length $(\mathrm{bp})$ & 114 \\
\hline Max length $(\mathrm{bp})$ & 6849 \\
\hline Average length $(\mathrm{bp})$ & 932.04 \\
\hline Total coding gene $(\mathrm{bp})$ & 2957373 \\
\hline Coding ratio $(\%)$ & 87.39103724 \\
\hline tRNA & 63 \\
\hline rRNA & 8 \\
\hline
\end{tabular}


TABLE II. KEGG CATEGORIES

\begin{tabular}{|c|c|c|}
\hline Type & Subgroup & $\begin{array}{c}\text { Gene } \\
\text { number }\end{array}$ \\
\hline \multirow{2}{*}{ Cellular Processes } & Cell growth and death & 12 \\
\hline & Transport and catabolism & 8 \\
\hline \multirow{2}{*}{$\begin{array}{l}\text { Environmental } \\
\text { Information } \\
\text { processing }\end{array}$} & Membrane transport & 98 \\
\hline & Signal transduction & 78 \\
\hline \multirow{4}{*}{$\begin{array}{l}\text { Genetic Information } \\
\text { Processing }\end{array}$} & Folding, sorting and degradation & 39 \\
\hline & Replication and repair & 68 \\
\hline & Transcription & 4 \\
\hline & Translation & 85 \\
\hline \multirow{12}{*}{ Metabolism } & $\begin{array}{l}\text { Metabolism of terpenoids and } \\
\text { polyketides }\end{array}$ & 49 \\
\hline & $\begin{array}{c}\text { Glycan biosynthesis and } \\
\text { metabolism }\end{array}$ & 32 \\
\hline & Lipid metabolism & 97 \\
\hline & $\begin{array}{l}\text { Xenobiotics biodegradation and } \\
\text { metabolism }\end{array}$ & 89 \\
\hline & Energy metabolism & 148 \\
\hline & Overview & 246 \\
\hline & $\begin{array}{c}\text { Metabolism of cofactors and } \\
\text { vitamins }\end{array}$ & 129 \\
\hline & Amino acid metabolism & 241 \\
\hline & Nucleotide metabolism & 94 \\
\hline & Carbohydrate metabolism & 210 \\
\hline & Metabolism of other amino acids & 48 \\
\hline & $\begin{array}{l}\text { Biosynthesis of other secondary } \\
\text { metabolites }\end{array}$ & 15 \\
\hline \multirow{5}{*}{ Organismal Systems } & Nervous system & 2 \\
\hline & Endocrine system & 23 \\
\hline & Environmental adaptation & 3 \\
\hline & Excretory system & 2 \\
\hline & Immune system & 2 \\
\hline
\end{tabular}

\section{Nucleotide SEQUence AcCession Number}

The annotated genome sequence of Acinetobacter sp. Y1 has been deposited in the GenBank database under accession no SRP080211.

\section{ACKNOWLEDGMENT}

This work was supported by the Key research and Development Program of Shanxi Province (201603D321010), International Cooperation Projects of Shanxi Province (201603D421040), National Key research and Development Program (2016YFB0600502) and Science and Technology Development of Shanxi Province (20140321013-04)

\section{REFERENCES}

[1] Chan, J.Z., et al., Defining bacterial species in the genomic era: insights from the genus Acinetobacter. Bmc Microbiology, 2012. 12(1): p. 1-11.

[2] Park, Y.S., et al., Extensively drug-resistant Acinetobacter baumannii : risk factors for acquisition and prevalent OXA-type carbapenemasesa multicentre study. International Journal of Antimicrobial Agents, 2010. 36(5): p. 430-435.

[3] Grosso, F., et al., Emergence of an extreme-drug-resistant (XDR) Acinetobacter baumannii carrying blaOXA-23 in a patient with acute necrohaemorrhagic pancreatitis. Journal of Hospital Infection, 2010. 75(1): p. 82-83.

[4] Gkorezis, P., et al., Draft genome sequence of Acinetobacter calcoaceticus strain GK1, a hydrocarbon-degrading plant growthpromoting rhizospheric bacterium. Genome Announcements, 2015. 3(4): p. 1-2.

[5] Bing, W. and D. Sun, Detection of NDM-1 carbapenemase-producing Acinetobacter calcoaceticus and Acinetobacter junii in environmental samples from livestock farms. Journal of Antimicrobial Chemotherapy, 2015. 70(2).

[6] Zhang, D., et al., Draft genome sequences of Acinetobacter harbinensis strain HITLi 7T, isolated from river water. Genome Announcements, 2015. 3(2).

[7] Gkorezis, P., et al., Draft genome sequence of Acinetobacter oleivorans PF1, a diesel-degrading and plant-growth-promoting endophytic strain isolated from poplar trees growing on a dieselcontaminated plume. Genome Announcements, 2015. 3(1).

[8] Ren, Y.X., Y. Lei, and L. Xian, The characteristics of a novel heterotrophic nitrifying and aerobic denitrifying bacterium, Acinetobacter junii YB. Bioresource Technology, 2014. 171: p. 1-9.

[9] Zhao, B., et al., Heterotrophic nitrogen removal by a newly isolated Acinetobacter calcoaceticus HNR. Bioresource Technology, 2010. 101(14): p. 5194-5200.

[10] Yao, S., et al., Heterotrophic nitrification and aerobic denitrification at low temperature by a newly isolated bacterium, Acinetobacter sp. HA2. Bioresource Technology, 2013. 139C(13): p. 80-86.

[11] Huang, X., et al., Ammonium removal by a novel oligotrophic Acinetobacter sp. Y16 capable of heterotrophic nitrification-aerobic denitrification at low temperature. Bioresource Technology, 2013. 146(10): p. 44-50.

[12] Zhao, B., et al., $\mathrm{N}_{2} \mathrm{O}$ and $\mathrm{N}_{2}$ production during heterotrophic nitrification by Alcaligenes faecalis strain NR. Bioresource Technology, 2012. 116(4): p. 379-385.

[13] Yang, L., et al., Nitrogen removal characteristics of a heterotrophic nitrifier Acinetobacter junii YB and its potential application for the treatment of high-strength nitrogenous wastewater. Bioresource Technology, 2015. 193: p. 227-233.

[14] Shi, Z., et al., Biological removal of nitrate and ammonium under aerobic atmosphere by Paracoccus versutus LYM. Bioresource Technology, 2013. 148C(7): p. 144-148.

[15] Brierley, E.D.R. and M. Wood, Heterotrophic nitrification in an acid forest soil: isolation and characterisation of a nitrifying bacterium. Soil Biology \& Biochemistry, 2001. 33(10): p. 1403-1409.

[16] Liu, Y., et al., Heterotrophic nitrogen removal by Acinetobacter sp. $\mathrm{Y} 1$ isolated from coke plant wastewater. Journal of Bioscience and Bioengineering, 2015. 120(5): p. 549-554.

[17] Song, Y.J., et al., Effect of carbon and nitrogen sources on nitrogen removal by a heterotrophic nitrification-aerobic denitrification strain Y1. Acta Sci. Circumst., 2013. 33(9): p. 2491-2497.

[18] Aziz, R.K., et al., The RAST Server: rapid annotations using subsystems technology. Bmc Genomics, 2008. 9(8): p. 75.

[19] Lagesen, K., et al., RNAmmer: consistent and rapid annotation of ribosomal RNA genes. Nucleic Acids Research, 2007. 35(9): p. $3100-$ 3108 .

[20] Lowe, T.M. and S.R. Eddy. tRNAscan-SE: a program for improved detection of transfer RNA genes in genomic sequence. in Nucleic Acids Res. 1997.

[21] Moir, J.W., et al., The biochemical characterization of a novel nonhaem-iron hydroxylamine oxidase from Paracoccus denitrificans GB17. Biochemical Journal, 1996. 319 ( Pt 3)(3): p. 823-7.

[22] Jetten, M.S.M., P.D. Bruijn, and J.G. Kuenen, Hydroxylamine metabolism in Pseudomonas PB16: involvement of a novel hydroxylamine oxidoreductase. Antonie Van Leeuwenhoek, 1997. 71(1-2): p. 69-74. 